\title{
Diffractive Physics in the Near Future
}

\author{
Gilvan A. Alves ${ }^{1}$ \\ Centro Brasileiro de Pesquisas Físicas, Rua Xavier Sigaud 150, 22290-180 Rio de \\ Janeiro - RJ, Brazil
}

\begin{abstract}
This paper summarize the present status and near term perspectives for Diffractive Physics at Tevatron. We describe the new detectors that are being installed around the CDF and $\mathrm{D} \varnothing$ interaction regions, and discuss the physics topics accessible in view of these upgrades.
\end{abstract}

\section{Introduction}

Diffraction is certainly one of the oldest subject in physics. In particle physics we have seen a big development in the second half of the last century [1]. More recently, with the discovery of Hard Diffraction by the UA8-Collaboration [2], we have had a significant development of Diffractive Physics both in theoretical and experimental points of view.

On the experimental side we have now many results coming from HERA (H1 and ZEUS) [3] and the Tevatron (CDF and DØ)[4] in a large number of topics including diffractive structure functions, diffractively produced jets, diffractive production of Heavy Flavors, etc.

On the theoretical side[5], a great deal of progress have been made in several issues, like factorization, diffractive structure functions, Large Rapidity Gap Survival Probability, etc.

Although the experimental results from HERA are showing a consistent improvement in the quality of data, the Tevatron results are still plagued by poor statistics. Not to mention that some processes, like Double Pomeron Exchange, have not been directly observed, and for most of the data sample, the kinematical information ( $\mathrm{t}$ and $\xi$ ) is still missing.

Despite of the theoretical progress for the unification of the soft and hard aspects of the strong interaction, we still need more experimental data to guide some of the theoretical development. It is important to stress that currently about $40 \%$ of the $p \bar{p}$ total cross section is due to the Pomeron exchange, and at higher energies this value can be even larger. In this sense, it is extremely important to have precise data from the physical region of the proton antiproton interaction at the Tevatron, to answer questions like: Is the Pomeron picture universal?, what are its hadronic characteristics?, Is it a glueball?, a dual object?, what is the diffractive contribution for the heavy flavor cross sections?, can the Higgs and Centauros be produced diffractively at Tevatron energies? and so on.

All of these questions lead to the proposal of new devices inserted at Tevatron. CDF reinstalled and improved its Roman Pots Spectrometer, and added a new 
Miniplug Calorimeter plus Beam Shower Counters. D $\varnothing$ added a new Forward Proton Detector (FPD).

Figure 1 shows the growing interest in diffractive physics. There is a clear growth in the number of papers on this subject in particle physics[6].

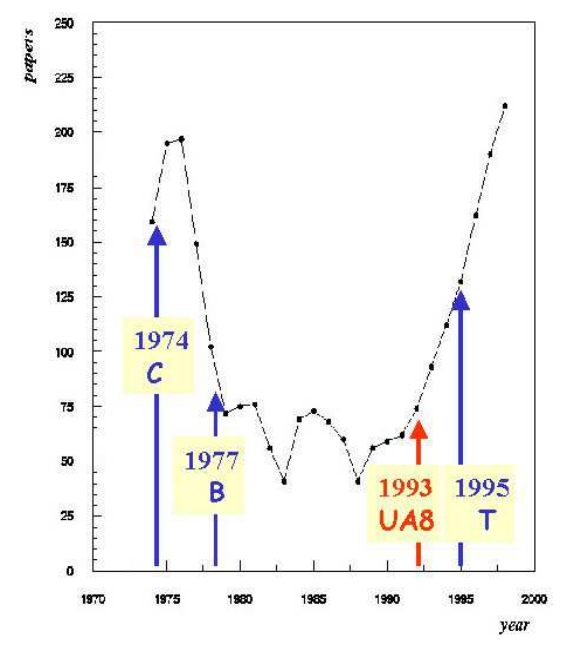

Fig. 1. Number of paper per year in diffractive physics. We show four important dates which have some correlation with diffractive physics papers. 1974 was the year of charm discovery, three years later, in 1977 the bottom, 1993 was the year of the hard diffraction discovery and 1995 the top discovery.

We are expecting a new period of diffractive results from several experiments in the near future. From the Tevatron, the upcoming Run II with CDF and D $\varnothing$; From Brookhaven National Laboratory $(B N L) /$ Relativistic Heavy Ion Collider $(R H I C)$ with the pp2pp experiment, polarized and unpolarized proton - proton interactions at energies of $\sqrt{s}=60-500 \mathrm{GeV}$; From the Deutsches Elecktronen Synchroton (DESY), with H1 and ZEUS, the two detectors at HERA, in electron (positron) $(27.5 \mathrm{GeV})$ proton $(820-920 \mathrm{GeV})$ interactions. For the far future we will have a new era of experiments, with TOTEM [7] being integrated into Compact Muon Solenoid (CMS) as one of the detectors of the Large Hadron Collider (LHC)/CERN, for proton - proton at $14 \mathrm{TeV}$ center of mass energy.

\section{The Tevatron Upgrades for Diffractive Physics CDF and $\mathrm{D} \varnothing$}

After the end of Run I, the Tevatron accelerator started its upgrade in order to achieve higher energy and luminosity. The main goals were to achieve energies up to $\sqrt{s}=2.0 \mathrm{TeV}$, and the luminosities of $\approx 3 \times 10^{32} \mathrm{~cm}^{-2} \mathrm{~s}^{-1}$. Besides, in 
order to implement the diffractive program at $D \emptyset$, some modifications had to be done to the accelerator itself. The two collider detectors CDF and D $\varnothing$ were also submitted to several upgrades, but we will restrict our discussion here only to those that have direct relation with diffractive physics.

The Tevatron beam line had to be modified to accommodate the new DØ leading proton spectrometers. This included modifying the electrostatic separator girder, extending the cryogenic bypass, removal of a quadrupole magnet $\left(Q_{1}\right)$, and other small modifications, like drilling a hole on the floor to allow the insertion and removal of detectors.

\section{$2.1 \quad \mathrm{CDF}$}

We will summarize the diffractive physics upgrades for CDF. For more details, please refer to the paper of Goulianos[8]. The CDF upgrades for diffractive physics can be divided in 3 parts:

1. Improve dipole spectrometer (Roman Pots) as is shown in figure 2

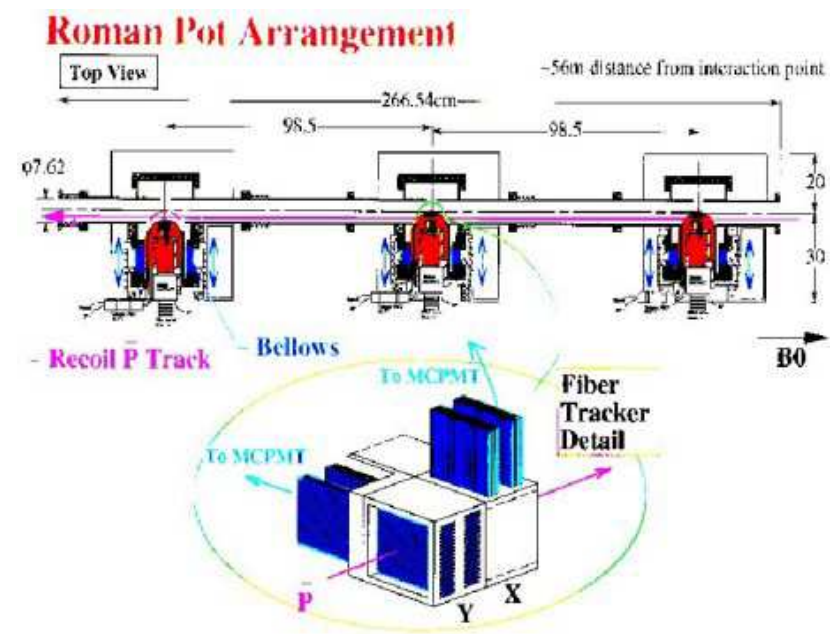

Fig. 2. Roman Pots arrangement of CDF.

2. Insertion of a Miniplug Calorimeter for triggering on diffractive events. This device, shown in figure 3 , is composed by $501 / 4$ " thick lead plates corresponding to a total of 2 interaction lengths and $\approx 60$ radiation lengths. 288 signal towers are viewed by 18 Multi Channel PMTs (16 channels each), covering a pseudorapidity region between 3.5 and 5.5.

3. Install new Beam Shower Counters at large rapidity region (5.5 - 7.5). These counters consist of regular plastic scintillators, read out by PMTs appropriate for high magnetic fields. 


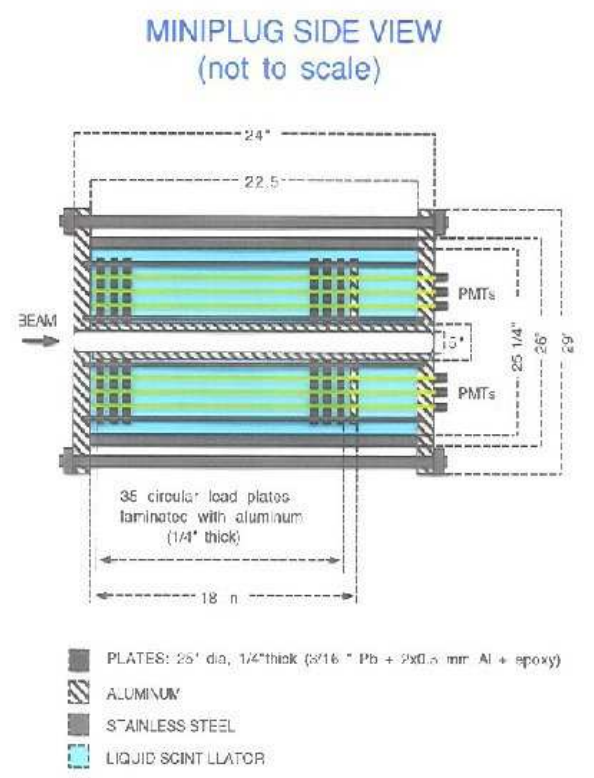

Fig. 3. Side view of the CDF Miniplug Calorimeter.

\section{$2.2 \quad \mathrm{D} \emptyset$}

The whole $\mathrm{D} \varnothing$ detector has been submitted to many modifications for the present run (RunII). Figure 4 summarize the main features.

Now we will describe the Forward Proton Detector, the main component of the D $\varnothing$ Diffractive Upgrade.

\section{Forward Proton Detector}

The idea of the Forward Proton Detector (FPD) is to cover experimentally a large number of topics which will be very important for the progress of diffractive physics. More details on the FPD can be read in the reference [9].

The Forward Proton Detector consists of 18 Roman Pots arranged on both sides of the $\mathrm{D} \emptyset$ detector as shown in figure 5, which shows the Roman Pot locations in the Tevatron beam line. There are two castles ${ }^{1}$ on the proton side indicated by $P 1$ and $P 2$ as shown in figure 5. The orientation is indicated by the additional letter $\mathrm{U}$ for up position, D for down position, I for inside position and $\mathrm{O}$ for outside position of the pots $(P 1 U, P 1 D, P 1 I, P 1 O$, same

\footnotetext{
${ }^{1}$ Structure that holds the Roman Pots, motors and vacuum equipment.
} 


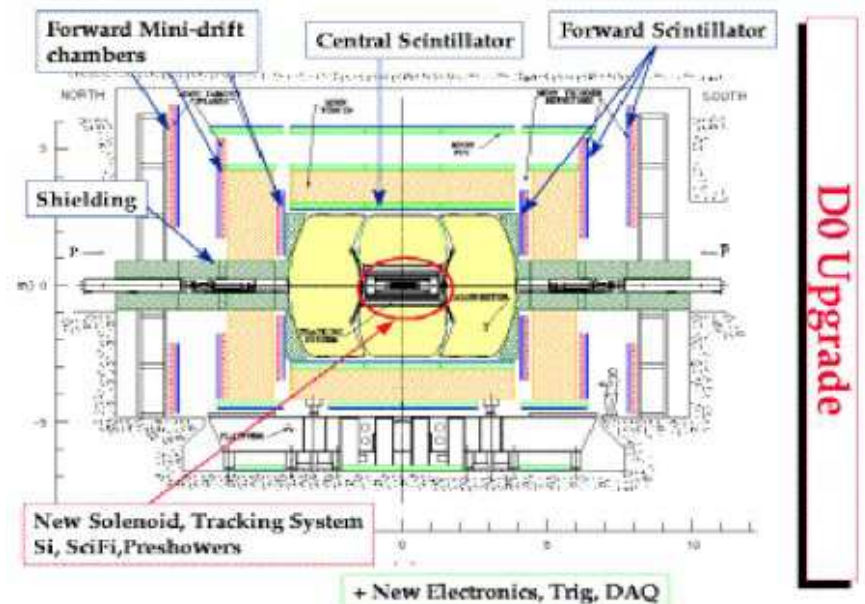

Fig. 4. The main components of the upgraded D $\varnothing$ detector for RunII.

notation for $P 2$ ). On the antiproton side we have two similar castles, labeled $A 1$ and $A 2$ followed by the indication of the orientation similar for the proton side. In addition, two others half castles on the antiproton side hold the dipole spectrometer ${ }^{2}$ labeled $D 1$ and $D 2$. The approximated distances of the pots with respect to the interaction point (indicated by 0 on the scale) are also shown.

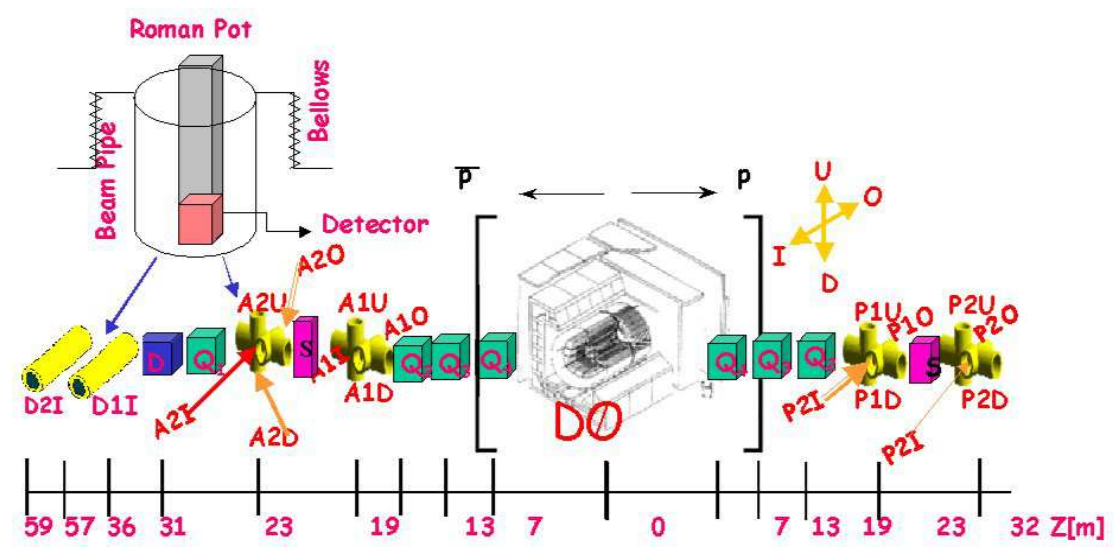

Fig. 5. This figure shows the FPD in the beam line of the Tevatron, in both sides of the D $\varnothing$ detector.

\footnotetext{
${ }^{2}$ Named in this way for their position after the dipole magnets.
} 


\subsection{Roman Pots}

The design of the Roman Pots for the DØ FPD is shown in figure 6. They were built by LNLS/Brazil (Laboratório Nacional de Luz Synchroton) as part of a regional collaboration ( LAFEX/CBPF - Centro Brasileiro de Pesquisas Físicas; UFBA - Universidade Federal da Bahia; UFRJ - Universidade Federal do Rio de Janeiro; UERJ - Universidade Estadual do Rio de Janeiro; IFT/UNESP Universidade Estadual Paulista; and LNLS) During two years we studied many options for the castle and detectors. The castle was made using 316L steel following the technical specifications to achieve a Ultra-High vacuum at the Tevatron. The combination of four view quadrupole stations, as shown in figure 6, and the dipole stations, give the possibility to cover a large portion of the available phase space, allowing for a better acceptance. In figure 6 we show the castle, indicating its main components. In order to have the best performance, the design of the pot window was submitted to a finite element analysis. The best results were obtained using a 150 microns foil, with elliptical cutout.

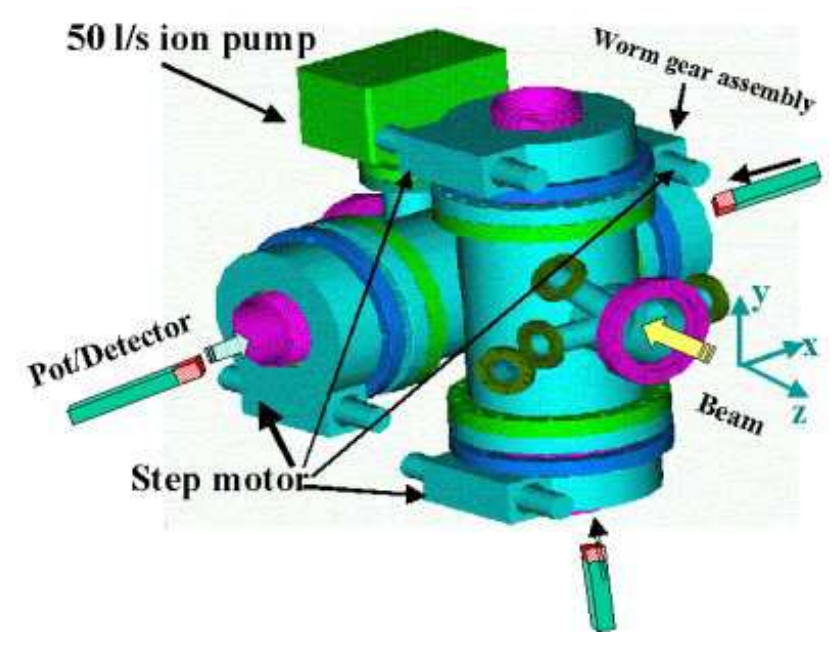

Fig. 6. This figure shows all main parts of the castle. The four "arms" can be seen, each one allowing for a pot to be inserted. The pot movement is done using step motors.

\subsection{The Detectors}

The FPD position detectors are based on square scintillating fibers, 800 micron thick, as shown in figure 7. In this figure we can see the frame of the scintillating fibers and 6 planes X X', U U' and V V' which compose one detector. The scintillating fibers are spliced (fused) to clear fibers which guide the signal up to the multi-anode photomultipliers (MAPMT H6568 from Hamamatsu). There are 
16 channels per X X' plane and 20 channels for the U U' and V V' planes, giving a total of 112 channels per detector and 2016 channels in total. Studies about the signal, efficiency and resolution have been made. Scintillating fibers are the best option for our detectors among many other possible technologies. The frame is made of polyurethane plastic. The theoretical resolution is 80 microns.

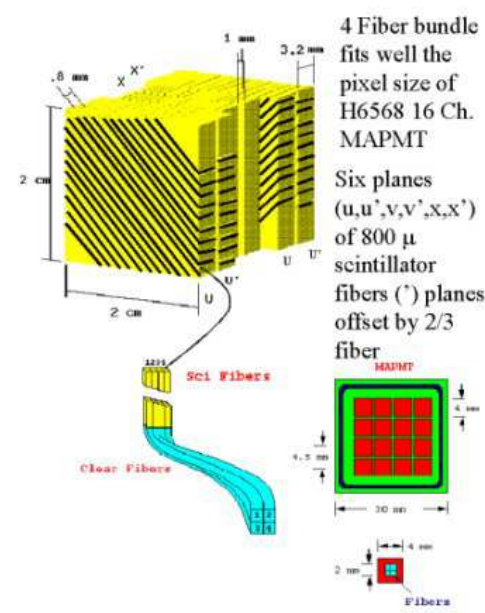

Fig. 7. This figure shows the six planes ( $\left.\mathrm{u} \mathrm{u}^{\prime}, \mathrm{v} \mathrm{v}^{\prime}, \mathrm{x} \mathrm{x}^{\prime}\right)$ of the detector for the FPD spectrometer. Four scintillating fibers are connected together in a single channel of the multi-anode photomultiplier (MAPMT).

The geometrical acceptance and the pot position acceptance are given in figure 8 .

\section{Some Physics Topics for the Run II at Tevatron}

The new Tevatron detectors were designed to cover a large region of phase space, allowing us to revisit some old and interesting results, and create conditions to observe new physics topics in diffraction. The combination of the Dipole and Quadrupole stations in the $\mathrm{D} \varnothing / \mathrm{FPD}$ will allow us to get a good sample of data. Early estimates on the size of this sample can be seen on table 1, where we also make a comparison with the available data to date.

In figure 9 we show several topologies available for study using this detector, some of them, like the Hard Double Pomeron Exchange, have only been studied indirectly using Rapidity Gap techniques.

We will now give a short description of some of the physics topics that can be studied with the new Tevatron Detectors: 


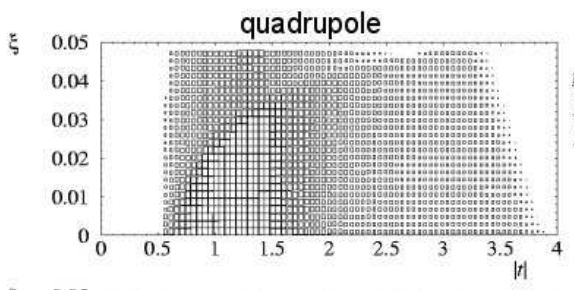

$\xi$
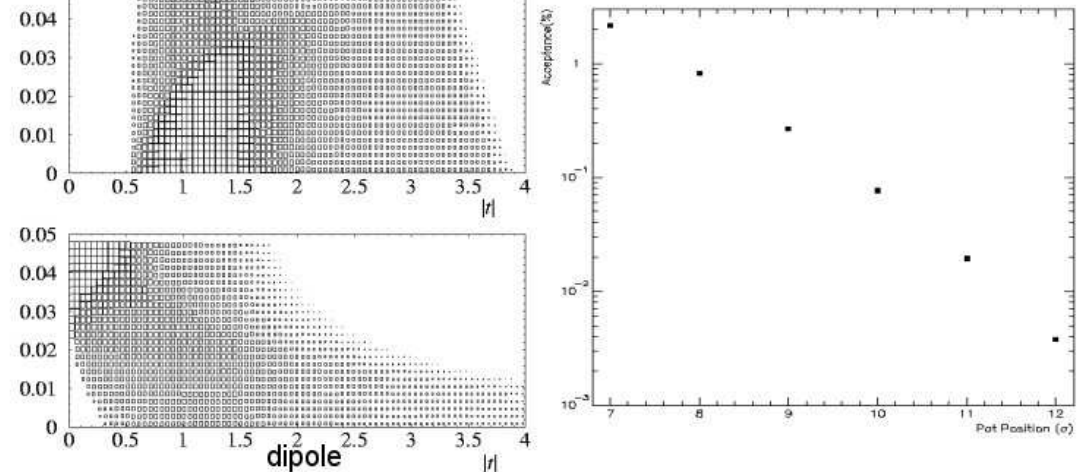

Fig. 8. This figure shows the $\xi$ and t acceptance for both dipole and quadrupole stations. The figure shows also the acceptance versus the pot position. We can see the acceptance variation by moving the pot as close as possible to the beam.

Table 1. This table shows a comparison between the present data on diffractive dijets and our expectation using the FPD at the Tevatron Run II.

\begin{tabular}{||c|c|c||}
\hline \hline Experiment & Dijet Events & $E_{T}[\mathrm{GeV}]$ \\
\hline \hline UA8 & 100 & 8 \\
\hline HERA & Hundreds & 5 \\
\hline CDF & Thousands & 10 \\
\hline \multirow{3}{*}{ D $\varnothing / F P D$} & 500,000 & 15 \\
\cline { 2 - 3 } & 150,000 & 20 \\
\cline { 2 - 3 } & 15,000 & 30 \\
\hline \hline
\end{tabular}

\subsection{Low and High $|t|$ Elastic Scattering}

The acceptance of the FPD spectrometers for low and high $\mathrm{t}$ will allow us to extract elastic scattering events in both of these physical regions. The measurements of elastic cross sections can be used to extract the total cross section via Optical Theorem. It is important to know the elastic slope of the differential cross section. The value of the slope for general differential cross sections characterizes a specific process which can be associated to a particular production (e.g. resonance production).

\subsection{Total Cross Section}

The results from Tevatron [10] experiments are not compatible between them, if we extrapolate to higher energies. It would be very important to have another measurement at these energies. It is important to know the behavior of the 

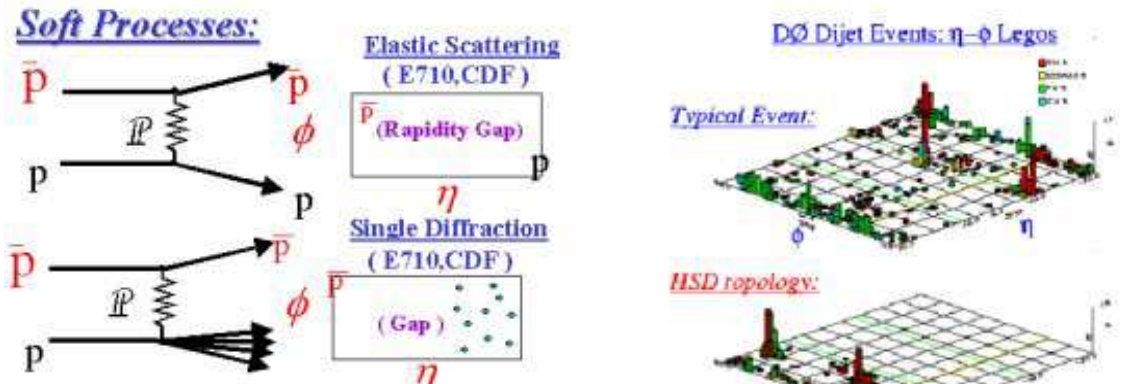

Hard Processes (jet production):

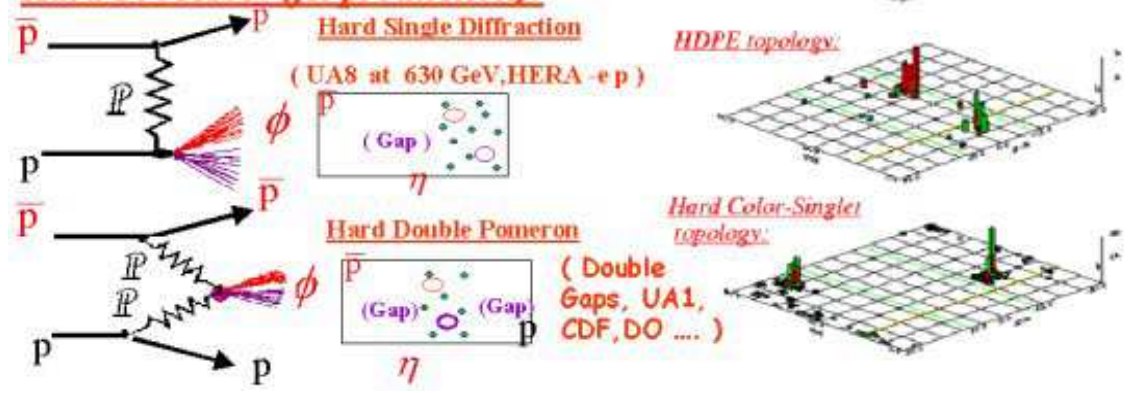

Fig. 9. This figure shows the possible topologies to be studied with FPD in the $\mathrm{D} \varnothing$ detector. For each topology we have a corresponding lego plot in pseudo-rapidity versus azimuthal angle. We also show the lego plots corresponding to 3 topologies: the Hard Single Diffraction (HSD), the Hard Double Pomeron Exchange (HDPE) and the Hard Color-Singlet extracted from DØ dijet events.

cross sections with energy since that is related to the Froissart-Martin bound. After the Tevatron only the LHC will offer a new opportunity to make these measurements.

\subsection{Inclusive Single Diffraction}

The inclusive single diffraction has many subtopics associated with it. Particularly for the Tevatron detectors, the Diffractive Mass available, for single diffraction events, $M_{x}=450 \mathrm{GeV}$, makes the extraction of heavy flavor physics very comfortable. Inclusive single diffraction has been a good laboratory for several problems in diffractive physics. In particular one would like to study the cross section dependence on the diffractive mass $\left(M_{x}\right)$ and four momentum transfered (t).

\subsection{Diffractive Jet production}

Jets have been largely studied by QCD. The discovery of diffractively produced jets [2] by UA8 collaboration was very important for diffractive physics. This 
was the main starting point for hard diffraction. It is expected that single and double diffractive jet production be exhaustively studied in the near future with the Tevatron and HERA detectors, making it possible to verify the universality of Diffractive Parton Distributions. As we see in table 1 it will be possible to get dijet events with higher transverse energies at the Tevatron Run II.

\subsection{Hard Double Pomeron Exchange}

Due to the interesting topology and exciting physics topics, double Pomeron exchange has been largely discussed as the process for producing many different types of states. [11]. An advantage of the large Diffractive Mass available at the Tevatron, in this case $M_{x} \simeq 100 \mathrm{GeV}$, is the possibility to study, by direct observation, the Pomeron $\times$ Pomeron interactions and the associated physics. The instrumentation available at $\mathrm{D} \varnothing$ is appropriated to face the challenges of the double Pomeron mechanism, and produce several objects not yet observed. Figure 9 shows the double Pomeron exchange graph and the gaps on its corresponding lego plot. Other topics like Glueballs, Centauros and Higgs can be exploited also in this topology.

\subsection{Diffractive Heavy Flavor Production}

Heavy Flavor Production, has been extensively studied in high $p_{t}$ physics. Because experimental results are scarce for diffractive heavy flavor production, there was not enough attention to this physics. For the lack of adequate instrumentation we have cross sections without a clear separation of diffractive and non-diffractive events. In principle we can split the studies of diffractive heavy flavor production based on the products: (i) The Charm, (ii) the Bottom and the (iii) Top Physics. Each one having some particularities to be taken into account in the diffractive production [12].

\subsection{Diffractive $\mathrm{W} / \mathrm{Z}$ boson production}

Diffractively produced $\mathrm{W}$ and $\mathrm{Z}$ bosons have been observed at the Tevatron. However the present results are not satisfactory, we need more statistics for these kind of events, to help us understand and set constraints on the quark and gluon contents of the Pomeron. Both CDF [13] and DØ have made progress, and the current results are motivating the collaborations to proceed with this measurements.

\subsection{Diffractive Structure Functions}

The study of Diffractive Structure Functions at the Tevatron allows a comparison with the existing HERA results. To understand the structure of the Pomeron one must know its structure function. This type of study has to be pursued exhaustively to get better and accurate measurements, building a clear interpretation 
of the Pomeron. One should be able to answer how important are the gluon and the quark components of the Pomeron. The universality of the Pomeron components is also important for this interpretation. Practically all diffractive topics mentioned here depend on this knowledge.

\subsection{Glueballs, Centauros and the Higgs}

Since the origin of QCD, Glueballs has been studied by theoreticians and experimentalists. However, we do not have a significant progress in this field. Glueballs are perfectly valid states in QCD, so if we do not find them, there must be some unknown suppression rule for this bound states. That means we need dedicated experiments to search for glueballs without ambiguity with quark anti-quark bound states. The family of glueballs is large. Table 2 shows this family (oddballs are also shown). Oddballs should have the priority to be examined, due to the fact that they can not be mistaken by $q \bar{q}$ (meson) or qqq (baryon) bound states with the same quantum numbers. It is a common belief that Glueballs should be largely produced in the double Pomeron Exchange topology.

Table 2. This table shows possible glueball state configurations with the mass and the quantum numbers for each one.

\begin{tabular}{|c|c|c|c|c|c|c|c|}
\hline \multicolumn{8}{|c|}{ Glueballs and Oddballs } \\
\hline \multirow[t]{2}{*}{$J^{P C}$} & \multirow[t]{2}{*}{$(q \bar{q})$} & \multirow[t]{2}{*}{$2 \mathrm{~g}$} & \multirow[t]{2}{*}{$3 \mathrm{~g}$} & \multirow[t]{2}{*}{ ODD } & \multicolumn{3}{|c|}{ MASS (GeV) } \\
\hline & & & & & [15] & {$[16]$} & [17] \\
\hline$\overline{0^{++}}$ & YES & YES & YES & $\mathrm{NO}$ & 1.58 & $1.73 \pm 0.13$ & $1.74 \pm 0.05$ \\
\hline$\overline{0}^{+-}$ & $\mathrm{NO}$ & $\mathrm{NO}$ & YES & YES & & & \\
\hline $0^{-+}$ & YES & YES & YES & $\mathrm{NO}$ & & & \\
\hline $0^{--}$ & $\mathrm{NO}$ & $\mathrm{NO}$ & YES & YES & 2.56 & $2.59 \pm 0.17$ & $2.37 \pm 0.27$ \\
\hline $1^{++}$ & YES & YES & YES & $\mathrm{NO}$ & & & \\
\hline $1^{+-}$ & YES & $\mathrm{NO}$ & YES & $\mathrm{NO}$ & & & \\
\hline $1^{-+}$ & $\mathrm{NO}$ & $\overline{Y E S}$ & YES & YES & & & \\
\hline $1^{-}$ & YES & $\mathrm{NO}$ & YES & $\mathrm{NO}$ & 3.49 & $3.85 \pm 0.24$ & \\
\hline $2^{++}$ & YES & YES & YES & $\mathrm{NO}$ & 2.59 & $2.40 \pm 0.15$ & $2.47 \pm 0.08$ \\
\hline $2^{+-}$ & $\mathrm{NO}$ & $\mathrm{NO}$ & YES & YES & & & \\
\hline $2^{-+}$ & YES & YES & YES & $\mathrm{NO}$ & 3.03 & $3.1 \pm 0.18$ & $3.37 \pm 0.31$ \\
\hline $2^{--}$ & YES & $\mathrm{NO}$ & YES & $\mathrm{NO}$ & 3.71 & $3.93 \pm 0.23$ & \\
\hline $3^{++}$ & YES & YES & YES & $\mathrm{NO}$ & 3.58 & $3.69 \pm 0.22$ & $4.3 \pm 0.34$ \\
\hline $3^{+-}$ & YES & NO & YES & $\mathrm{NO}$ & & & \\
\hline $3^{-+}$ & $\mathrm{NO}$ & YES & YES & YES & & & \\
\hline $3^{--}$ & YES & $\mathrm{NO}$ & YES & $\mathrm{NO}$ & 4.03 & $4.13 \pm 0.29$ & \\
\hline
\end{tabular}


Another topic is the production of Centauros, which were never observed in accelerator experiments. These objects were discovered in Cosmic Ray Physics as events with several unusual characteristics, like the production of a large multiplicity of charged particles, accompanied by very few photons. For example, as many as 100 charged particles and no more than $3 \pi^{0}$. [14] There is enough center of mass energy at the Tevatron to produce Centauros, and since the diffractive mass is high enough, it is possible to produce them diffractively. The good calorimetry of the $\mathrm{D} \varnothing$ detector can be very useful in observing this kind of events.

Higgs is one of the most exciting subjects for the Tevatron Run II. It has not been excluded the possibility that it can be produced also diffractively. We have two recent studies given by reference [11], showing the possibility of Higgs production by the double Pomeron mechanism.

\section{Conclusions}

There is a lot of exciting new results coming from the investigation of diffractive phenomena, in particular the ongoing Run II at Tevatron should give us a better picture of diffraction with the new detectors at CDF and D $\varnothing$. Practically all possible subjects listed here can be studied with the data obtained from these detectors.

The main goal of the experiments in diffractive physics is hard diffraction. Many theoretical and phenomenological progress have been made with the precise data from HERA, but a corresponding set of data from the Tevatron is still missing to allow comparisons between the two regimes of production. This set of data will give us the opportunity to test assumptions like the universality of the Pomeron picture and the Diffractive Parton Distributions.

Another important topic concerns the measurement of the Total Cross Section at the highest energies of the Tevatron, for which there are conflicting results that can be settled by a new measurement from the FPD at DØ. This new measurement will also help to reduce uncertainties on the luminosity for all DØ physics processes.

The double Pomeron exchange and the physics that can be done with this topology is one of the very important subjects of this run at the Tevatron. It has not been excluded, as we called attention, that Centauros and Higgs [11] can be produced diffractively in this topology.

Finally the diffractive physics results from $\mathrm{CDF}$ and $\mathrm{D} \emptyset$ will be very important for future projects at the LHC, since it is expected that the diffractive contribution becomes more important at increasing energies.

I would like to thank the organizing committee for the superb job in the organization a very enjoyable meeting. In particular I would like to thank Profs. J. Trampetic and J. Wess for the kind invitation and financial support. 


\section{References}

1. V. Barone and E. Predazzi, High Energy Particle Diffraction, Springer, (2001).

2. A. Brandt et al., UA8 Collaboration, Nucl. Inst. Meth.A327, 412 (1993); ibid., Phys. Lett. B297, 93 (1993); ibid., HEP-Ex-9709015, 1997, submitted to Phys. Lett. B.

3. ZEUS collaboration, M. Derrick et.al., Z. Phys. C65, 379,(1995); H1 collaboration, T.Ahmed et.al., Nucl. Phys. B439, 471 (1995); ZEUS collaboration, M. Derrick et.al. Z. Phys. C68, 569, (1995); H1 collaboration, T.Ahmed et.al. Phys. Lett. B348, $681(1995)$;

4. G.A. Alves, Diffractive Results from the Tevatron, Proceedings of DPF99, Los Angeles (1999) hep-ex/9905009; CDF Collab., T. Affolder et al., Phys. Rev. Lett. 84, 5043 (2000).

5. For a nice review see G.Iacobucci, Invited talk at 20th International Symposium on Lepton and Photon Interactions at High Energies (Lepton Photon 01) hepex/0111079

6. E. M. Gregores, T.L. Lungov and S. F. Novaes, A.Santoro, Proceedings of Hadron 2000 - S. Paulo - Brazil (2000).

7. G. Matthiae, The Future of Diffraction at the LHC, Proceedings of DIFFRACTION 2000, Nucl.Phys.Proc.Suppl.99A 281-288 (2001).

8. K. Goulianos, Diffraction in CDF: Run I Results and Plans for Run II, Proceedings of DIS 2001, hep-ex/0107069 (2001).

9. A. Brandt et al. A Forward Proton Detector Fermilab - Pub-97/377, 1997

10. C. Avila, Proceedings of LISHEP98; Thesis: Measurement the proton - antiproton total cross section at center of mass Energy of $1800 \mathrm{GeV}$, Cornell University 1997 and references therein; C. Avila et al., Phys. Lett. B234, 158, (1990);ibid., Phys. Rev. Lett. 68, 2433, (1992); ibid., Phys. Rev. 50, 5550, (1994); ibid., Phys. Lett. B234, 158, (1990); ibid., Nucl. Inst. Meth.A360, 80, (1995)

11. See for example for Higgs production the paper of D.Kharzeev and E. Levin, Soft Double-Diffractive Higgs Production at Hadron Collider., Fermilab-Pub-00/035T; BNL-NT-00/14; Hep-ph/0005311 (2000); and V.A.Khoze, A.D.Martin and M.G.Ryskini, Can the Higgs be seen in rapidity gap events at the Tevatron or the LHC?, DTP/00/08; hep-ex/0002072 (2000)

12. E.L.Berger, J.C.Collins, D.E. Soper, G.Sterman, Nucl. Phys. B286, 704, (1987); A. Kerman and G.Van Dalen, Phys. Rep. 106, 297, (1984)

13. F.Abe et al. CDF Collaboration: Phys. Rev. Lett. 78, 2698, (1997)

14. F. Halzen, FELIX: The Cosmic Ray Connection, Felix Home Page, http://felix.web.cern.ch/FELIX/Physics/paperi.html, Brazil-Japan Collaboration Proceedings of the 21st. Int.Conf.-Adelaide-Australia vol.8,259 (1990); C. M. G. Lattes, Y. Fujimoto and S. Hasegawa, Phys. Rep. 65, 151, 1980; C. E. Navia et al.: Phys. Rev D40, 2898, (1989)

15. A. B. Kaidalov and Yu. A. Simonov, Glueball masses and Pomeron Trajectory in non perturbative QCD approach, hep-ph/9912434 (1999)

16. C. Morningstar, M. Peardor, Nucl. Phys. B63A-C, 1022 (1998); Phys. Rev. D60,034509 (1999)

17. M. Teper, Glueball masses and other physical properties of $S U(N)$ gauge theories in $D=3+1$ : a review of lattice results for theorists, Hep/th981287 (1998) 